\title{
Risk factors for overcorrection of severe hyponatremia: a post hoc analysis of the SALSA trial
}

\author{
Huijin Yang ${ }^{1}$, Songuk Yoon ${ }^{1}$, Eun Jung Kim¹, Jang Won Seo ${ }^{1}$, Ja-Ryong $\mathrm{Koo}^{1}$, Yun Kyu $\mathrm{Oh}^{2}$, You Hwan Jo ${ }^{3}$, \\ Sejoong Kim ${ }^{4,5,6}$, Seon Ha Baek ${ }^{1}$
}

${ }^{1}$ Division of Nephrology, Department of Internal Medicine, Hallym University Dongtan Sacred Heart Hospital, Hwaseong, Republic of Korea

${ }^{2}$ Division of Nephrology, Department of Internal Medicine, SMG-SNU Boramae Medical Center, Seoul, Republic of Korea

${ }^{3}$ Department of Emergency Medicine, Seoul National University Bundang Hospital, Seongnam, Republic of Korea

${ }^{4}$ Division of Nephrology, Department of Internal Medicine, Seoul National University Bundang Hospital, Seongnam, Republic of Korea

${ }^{5}$ Center for Artificial Intelligence in Healthcare, Seoul National University Bundang Hospital, Seongnam, Republic of Korea

${ }^{6}$ Department of Internal Medicine, Seoul National University College of Medicine, Seoul, Republic of Korea

\begin{abstract}
Background: Hyponatremia overcorrection can result in irreversible neurologic impairment such as osmotic demyelination syndrome. Few prospective studies have identified patients undergoing hypertonic saline treatment with a high risk of hyponatremia overcorrection.

Methods: We conducted a post hoc analysis of a multicenter, prospective randomized controlled study, the SALSA trial, in 178 patients aged above 18 years with symptomatic hyponatremia (mean age, 73.1 years; mean serum sodium level, $118.2 \mathrm{mEq} / \mathrm{L}$ ). Overcorrection was defined as an increase in serum sodium levels by $>12$ or $18 \mathrm{mEq} / \mathrm{L}$ within 24 or 48 hours, respectively.

Results: Among the 178 patients, 37 experienced hyponatremia overcorrection (20.8\%), which was independently associated with initial serum sodium level $(\leq 110,110-115,115-120$, and $120-125 \mathrm{mEq} / \mathrm{L}$ with $7,4,2$, and 0 points, respectively), chronic alcoholism ( 7 points), severe symptoms of hyponatremia ( 3 points), and initial potassium level ( $<3.0 \mathrm{mEq} / \mathrm{L}, 3$ points). The NASK (hypoNatremia, Alcoholism, Severe symptoms, and hypoKalemia) score was derived from four risk factors for hyponatremia overcorrection and was significantly associated with overcorrection (odds ratio, 1.41; 95\% confidence interval, 1.24-1.61; $p<0.01$ ) with good discrimination (area under the receiver-operating characteristic [AUROC] curve, $0.76 ; 95 \% \mathrm{Cl}, 0.66-0.85 ; p<0.01$ ). The AUROC curve of the NASK score was statistically better compared with those of each risk factor.

Conclusion: In treating patients with symptomatic hyponatremia, individuals with high hyponatremia overcorrection risks were predictable using a novel risk score summarizing baseline information.
\end{abstract}

Keywords: Hyponatremia, Novel risk score, Overcorrection, Prediction, Risk factors

Received: August 14, 2021; Revised: November 16, 2021; Accepted: November 21, 2021

Correspondence: Seon Ha Baek

Division of Nephrology, Department of Internal Medicine, Hallym University Dongtan Sacred Heart Hospital, 7 Keunjaebong-gil, Hwaseong 18450, Republic of Korea. E-mail: seonhabaek@hallym.or.kr, haya2001@hanmail.net

ORCID: https://orcid.org/0000-0002-4751-9817

\section{Sejoong Kim}

Division of Nephrology, Department of Internal Medicine, Seoul National University Bundang Hospital, 82 Gumi-ro 173beon-gil, Bundang-gu, Seongnam 13620, Republic of Korea. E-mail: sejoong@snubh.org

ORCID: https://orcid.org/0000-0002-7238-9962

Seon Ha Baek and Sejoong Kim contributed equally to this study as co-corresponding authors.

Copyright @ 2022 by The Korean Society of Nephrology

(a) This is an Open Access article distributed under the terms of the Creative Commons Attribution Non-Commercial and No Derivatives License (http:// creativecommons.org/licenses/by-nc-nd/4.0/) which permits unrestricted non-commercial use, distribution of the material without any modifications, and reproduction in any medium, provided the original works properly cited. 


\section{Introduction}

Hyponatremia is the most common electrolyte imbalance encountered in clinical practice, with a prevalence of $14 \%$ to $42 \%$ in hospitalized patients. Moreover, hyponatremia has a high mortality rate and long hospitalization period $[1,2]$. Hyponatremia can induce various clinical manifestations that range from mild (fatigue, nausea, vomiting, headache, gait disorder, and confusion) to severe symptoms (seizures, coma, and brain hypoxia) [3-6]. Hypertonic saline has been used to treat symptomatic hyponatremia $[7,8]$. An increase in serum sodium levels by 4 to $6 \mathrm{mEq} / \mathrm{L}$ is generally sufficient to improve the symptoms caused by cerebral edema. However, overcorrection of hyponatremia may result in irreversible neurologic disability such as osmotic demyelination syndrome (ODS) [9-12].

According to an American expert panel recommendation, overcorrection is defined as increase in serum sodium levels of $>10-12 \mathrm{mEq} / \mathrm{L}$ in any 24 hours period or $>18 \mathrm{mEq} /$ $\mathrm{L}$ in any 48 hours period, with a more stringent limit of $>8$ $\mathrm{mEq} / \mathrm{L}$ in 24 hours for patients at a high risk of developing ODS [13]. A European clinical practice guideline defined overcorrection as an increase in serum sodium levels of $>10 \mathrm{mEq} / \mathrm{L}$ during the first 24 hours or $>8 \mathrm{mEq} / \mathrm{L}$ in any 24 hours period thereafter [1]. The distinction between both guidelines indicates that there is still no consensus regarding the definition of hyponatremic overcorrection $[1,10,13-$ 17]. Nevertheless, the incidence of overcorrection has been reported to be as high as $20 \%$ to $41 \%$ [10,14,18-21].

Several studies and guidelines have recommended a specific amount and rate of hypertonic saline for effective hyponatremia treatment and overcorrection prevention $[1,10,13,16,18,20,22-24]$. However, it remains unclear why overcorrection occurs in some patients that received the required amount of hypertonic saline at the recommended rate. A few studies have examined the risk factors for overcorrection following symptomatic hyponatremia treatment, and these studies were limited by their retrospective design $[14,17,18,21]$. Therefore, we conducted a post hoc analysis of a prospective randomized controlled study, in which we investigated risk factors for overcorrection in patients receiving treatment for severe hyponatremia. Our goal was to enable physicians to recognize individuals at a high risk of overcorrection and to prevent ODS by careful correction with hypertonic saline.

\section{Methods}

\section{Study population}

We performed a post hoc analysis of a multicenter, prospective randomized controlled study: the SALSA (Efficacy and Safety of Rapid Intermittent Correction Compared With Slow Continuous Correction With Hypertonic Saline In Patients With Moderately Severe or Severe Symptomatic Hyponatremia) trial. The study included 178 participants who were admitted at Seoul National University Bundang Hospital, SMG-SNU Boramae Medical Center, and Hallym University Dongtan Sacred Heart Hospital between August 2016 and August 2019. The detailed study protocol has been described elsewhere (ClinicalTrials.gov; NCT02887469) [19].

Patients aged above 18 years, with moderate to severe symptoms, and with glucose-corrected serum sodium levels of $\leq 125 \mathrm{mEq} / \mathrm{L}$ were included in this study [25]. Patients with the following conditions were excluded: pseudohyponatremia (serum osmolality, $>275 \mathrm{mOsm} / \mathrm{kg}$ ), primary polydipsia (urine osmolality, $\leq 100 \mathrm{mOsm} / \mathrm{kg}$ ), current pregnancy, breastfeeding, anuria, arterial hypotension (systolic blood pressure, $<90 \mathrm{mmHg}$ and mean arterial pressure, $<70 \mathrm{mmHg}$ ), liver disease (transaminase levels of $>$ three times the upper limit of normal, known decompensated liver cirrhosis with ascites or diuretic use, hepatic encephalopathy, and esophageal varices), uncontrolled diabetes mellitus (glycated hemoglobin, $>9$ ), a history of cardiac surgery, acute myocardial infarction, sustained ventricular tachycardia, ventricular fibrillation, acute coronary syndrome, cerebral trauma, and increased intracranial pressure. This post hoc analysis was approved by the Institutional Review Boards of three centers: Seoul National University Bundang Hospital (No. B-2101-660-101), SMGSNU Boramae Medical Center (No. 10-2021-6), and Hallym University Dongtan Sacred Heart Hospital (No. 2020-10012). Written consent was obtained from all participants or a legal guardian, when applicable. The study complied with principles of the Declaration of Helsinki.

\section{Data collection and definitions}

Baseline demographics, alcohol consumption information, and anthropometric measurements were recorded. Chron- 
ic alcoholism was defined as the consumption of at least 4 and 3 glasses of alcohol per day in males and females, respectively, regardless of the types of alcohol. We assessed the presence of comorbidities such as hypertension, diabetes mellitus, congestive heart failure, and cancer by screening for the I10-I15; E10-14; I11.0, I13.0, I13.2, I50; and C codes based on the International Classification of Disease, the 10th revision as well as by a self-reported or confirmed history of antihypertensive and antidiabetic drug use. The definition of hyponatremia was based on biochemical severity: 'severe (profound)' was defined as serum sodium levels of $\leq 125 \mathrm{mEq} / \mathrm{L}[1,13]$. Clinical manifestations of hyponatremia were divided into moderate and severe based on the clinical presentation of the patient at initial hyponatremia. Moderate symptoms included nausea, drowsiness, headache, general weakness, and malaise. Severe symptoms included vomiting, stupor, coma (Glasgow Coma Scale score, $\leq 8$ ), and seizures. We determined the underlying cause of hyponatremia using a structured diagnostic approach based on history, physical examination, and laboratory test findings. Patients were divided into five categories: 1) decreased extracellular fluid (ECF) volume due to renal sodium loss (e.g., diuretics, especially thiazides), 2) decreased ECF volume due to nonrenal sodium loss (e.g., gastrointestinal sodium loss or third spacing), 3) increased ECF volume (e.g., heart failure, liver cirrhosis, and nephrotic syndrome), 4) normal ECF volume with adrenal insufficiency, and 5) normal ECF volume fulfilling essential diagnostic criteria for the syndrome of inappropriate antidiuresis (SIAD) [20,26].

Two infusion methods for hypertonic saline have been described in published protocols; rapid intermittent bolus (RIB) and slow continuous infusion (SCI) $[19,20,27]$. The initial infusion rate was determined based on hyponatremia symptom severity. The treatment guidelines for the two groups are detailed in Supplementary Methods (available online) and Supplementary Fig. 1 (available online). The treatment goals were to increase serum sodium level by $5-9 \mathrm{mEq} / \mathrm{L}$ and achieve symptom relief within the first 24 hours, as well as increase serum sodium level by $10-17$ $\mathrm{mEq} / \mathrm{L}$ or to $\geq 130 \mathrm{mEq} / \mathrm{L}$ and to achieve symptom relief within the first 48 hours. Serum sodium levels were measured every 6 hours for 2 days using indirect ion-selective electrodes at the following three centers with the indicated equipment: Seoul National University Bundang Hospital,
AU5800 (Beckman Coulter, Indianapolis, IN, USA) and Dimension Vista 1500 (Siemens Healthineers, Erlangen, Germany); SMG-SNU Boramae Medical Center, Modular DP (Roche Diagnostics, Indianapolis, IN, USA) and Unicel DxC 800 (Beckman Coulter); and Hallym University Dongtan Sacred Heart Hospital, AU5800 (Beckman Coulter).

\section{Study outcomes}

The primary outcome was the incidence of hyponatremia overcorrection at any given period, which was defined as an increase in serum sodium levels by $>12 \mathrm{mEq} / \mathrm{L}$ or $>18$ $\mathrm{mEq} / \mathrm{L}$ within the first 24 or 48 hours, respectively. The secondary outcomes represented the time-specific increase in cumulative hyponatremia overcorrection rates specified by time. These were defined as an increase in serum sodium levels by $>12 \mathrm{mEq} / \mathrm{L}$ within 6,12 , and 24 hours.

\section{Statistical analysis}

Baseline characteristics and laboratory data are expressed as mean and standard deviation for continuous variables and frequency and percentage for discrete variables. Differences in continuous variables were analyzed using the Student $\mathrm{t}$ test and Mann-Whitney test, and the chi-square and Fisher exact tests were used for discrete variables. Univariable logistic regression was used to analyze each variable to identify significant risk factors for hyponatremia overcorrection. We retained variables with $\mathrm{p}<0.05$ in the multivariable model using backward selection. Odds ratios (ORs) and 95\% confidence intervals (CIs) for hyponatremia overcorrection occurrence were calculated after stepwise adjustment for multiple confounders. We multiplied parameter estimates of discrete variables in the model by a constant to obtain scores. We multiplied coefficients of continuous variables by the constant, which then represented a risk score for each unit increase in an individual continuous variable. The risk score was summarized as the arithmetic sum of the points for each variable. We evaluated model discrimination using the area under the receiver-operating characteristic (AUROC) curve to compare our predictive model to an older overcorrection model (The Severe Hyponatremia Overcorrection Risk [SHOR] score) [17]. The SHOR score has eight risk factors for overcorrection: age, vomiting, somnolence, volume overload, initial 
serum sodium level, initial serum potassium level, urine osmolality, and chest tumor. We adjusted our prospective data to compare the predictive abilities for overcorrection in the SHOR scoring system and our predictive model. Patients aged under 40 years were included in the 40 to 50 years group, and those with serum sodium levels of $>116$ $\mathrm{mEq} / \mathrm{L}$ were included in the serum sodium of 114-116 $\mathrm{mEq} / \mathrm{L}$ group. We defined somnolence as drowsiness or stupor. We obtained two scores (SHOR1 score: somnolence as stupor, SHOR2 score: somnolence as drowsiness), and each SHOR score was compared with that of our predictive model. Variables with $\mathrm{p}<0.05$ were considered statistically significant. All analyses and calculations were performed using IBM SPSS version 24.0 (IBM Corp., Armonk, NY, USA), and STATA version 14.0 (StataCorp LP, College Station, TX, USA).

\section{Results}

\section{Study population}

We enrolled 178 patients who were admitted for symptomatic hyponatremia between August 24, 2016 and August 21,2019 . The mean patients' age was $73.1 \pm 12.2$ years and $44.9 \%$ of the patients were male. The most common causes of hyponatremia were thiazide diuretic use ( $\mathrm{n}=53$ [29.8\%]), and SIAD ( $\mathrm{n}=52$ [29.2\%]), with almost the same incidence. These were followed by adrenal insufficiency (n $=44$ [24.7\%]), decreased ECF volume due to nonrenal sodium loss ( $\mathrm{n}=39[21.9 \%])$, and increased ECF volume $(\mathrm{n}=19$ [10.7\%]). Five individuals had a history of chronic alcoholism $(n=5[2.8 \%])$. Forty-four patients $(24.7 \%)$ had severe symptoms of hyponatremia. Serum sodium and potassium levels at admission were $118.2 \pm 5.0$ and $4.0 \pm 0.8 \mathrm{mEq} / \mathrm{L}$, respectively.

Incidence and risk factors for hyponatremia overcorrection

Hyponatremia overcorrection occurred in $20.8 \%$ (37 of 178) of patients. Patients were classified into overcorrection and no-overcorrection groups, and their baseline characteristics are shown in Table 1. Patients in the overcorrection group were more likely to exhibit chronic alcoholism (8.1\% vs. $1.4 \%$ ), severe symptoms ( $45.9 \%$ vs. $19.1 \%$ ), and thiazide use ( $43.2 \%$ vs. $26.2 \%$ ) than those in the no-overcorrection group. The overcorrection group demonstrated lower levels of serum sodium, potassium phosphorus, and osmolarity compared with the no-overcorrection group. The overcorrection group also showed higher serum albumin, aspartate aminotransferase, alanine aminotransferase, and total bilirubin levels than those of the no-overcorrection group. The cumulative amount of hypertonic saline administrated during 48 hours was $554 \mathrm{~mL}$. The cumulative amounts of hypertonic saline infused during the first $1 / 6$ hours did not differ between the groups. Interestingly, the cumulative amounts of hypertonic saline administrated for 24/48 hours were significantly smaller in the overcorrection group than that that in the no-overcorrection group (24 hours, $350 \mathrm{~mL}$ vs. $416.3 \mathrm{~mL}, \mathrm{p}=0.045 ; 48$ hours, 388.5 $\mathrm{mL}$ vs. $598.1 \mathrm{~mL}, \mathrm{p}<0.001)$. The 48 hours urine output was higher in the overcorrection than that in the no-overcorrection group $(5,663 \mathrm{~mL}$ vs. 3,401 $\mathrm{mL}, \mathrm{p}<0.001)$.

We conducted univariable and multivariable logistic regression analyses separately to identify independent risk factors for hyponatremia overcorrection (Table 2). The univariate analysis showed that chronic alcoholism (OR, 6.13; 95\% CI, 0.99- 38.16; $\mathrm{p}=0.05$ ), severe symptoms of hyponatremia (OR, 3.59; 95\% CI, 1.66-7.76; p < 0.01), low serum potassium level (OR, 0.37; 95\% CI, 0.21-0.65; $\mathrm{p}<0.01$ ), low serum sodium level (OR, 0.84; 95\% CI, 0.77-0.90; $\mathrm{p}<0.01$ ), high total bilirubin level (OR, 3.06; 95\% CI, 1.42-6.58; p < 0.01), high albumin level (OR, 2.38; 95\% CI, 1.19-4.76; p $=0.02)$, and thiazide use (OR, 2.14; 95\% CI, 1.01-4.54; $\mathrm{p}=$ $0.05)$ were significant risk factors for overcorrection. Multivariate analysis showed that only chronic alcoholism (OR, 15.27; 95\% CI, 1.46-159.28; $\mathrm{p}=0.02$ ), severe symptoms of hyponatremia (OR, 2.83; 95\% CI, 1.14-7.02; $\mathrm{p}=0.03$ ), initial serum potassium level (OR, 0.86; 95\% CI, 0.79-0.94; $\mathrm{p}$ $<0.01$ ), and initial serum sodium level (OR, 0.34; 95\% CI, $0.17-0.67 ; \mathrm{p}<0.01$ ) were significant risk factors for overcorrection after adjusting for all variables.

\section{Novel risk score for hyponatremia overcorrection (NASK score)}

We created a scoring system to facilitate the manual calculation of hyponatremia overcorrection risk, and we used coefficients to change each impact to integer scores for four statistically significant variables. We calculated the scores 
Table 1. Baseline characteristics and progression according to overcorrection

\begin{tabular}{|c|c|c|c|c|}
\hline Characteristics and progression & Total & No overcorrection & Overcorrection & p-value \\
\hline No. of patients & 178 & 141 & 37 & \\
\hline Male sex & $80(44.9)$ & 65 (46.1) & $15(40.5)$ & 0.55 \\
\hline Age $(y r)$ & $73.1 \pm 12.2$ & $73.7 \pm 11.8$ & $70.5 \pm 13.4$ & 0.23 \\
\hline Weight (kg) & $57.1 \pm 11.6$ & $56.7 \pm 11.4$ & $58.6 \pm 12.4$ & 0.58 \\
\hline Body mass index $\left(\mathrm{kg} / \mathrm{m}^{2}\right)$ & $22.6 \pm 4.3$ & $22.4 \pm 3.9$ & $23.3 \pm 5.3$ & 0.67 \\
\hline \multicolumn{5}{|l|}{ Causes of hyponatremia } \\
\hline Decreased ECF d/t nonrenal Na loss & $39(21.9)$ & $32(22.7)$ & $7(18.9)$ & 0.62 \\
\hline Increased ECF & $19(10.7)$ & $17(12.1)$ & $2(5.4)$ & 0.37 \\
\hline SIAD & $52(29.2)$ & $43(30.5)$ & $9(24.3)$ & 0.46 \\
\hline Thiazide use & $53(29.8)$ & $37(26.2)$ & $16(43.2)$ & 0.07 \\
\hline Adrenal insufficiency & $44(24.7)$ & $36(25.5)$ & $8(21.6)$ & 0.68 \\
\hline \multicolumn{5}{|l|}{ Comorbidity } \\
\hline Diabetes mellitus & $59(33.1)$ & $46(32.6)$ & $13(35.1)$ & 0.77 \\
\hline Hypertension & $123(69.1)$ & $93(66.0)$ & $30(81.1)$ & 0.08 \\
\hline Congestive heart failure & $31(17.4)$ & $25(17.7)$ & $6(16.2)$ & 0.83 \\
\hline Liver cirrhosis & $11(6.2)$ & $8(5.7)$ & $3(8.1)$ & 0.70 \\
\hline Nephrotic syndrome & $4(2.2)$ & $4(2.8)$ & $0(0)$ & 0.58 \\
\hline Hypothyroidism & $18(10.1)$ & $15(10.6)$ & $3(8.1)$ & 0.77 \\
\hline Malignancy & $42(23.6)$ & $35(24.8)$ & 7 (18.9) & 0.45 \\
\hline Chronic alcoholism & $5(2.8)$ & $2(1.4)$ & $3(8.1)$ & 0.06 \\
\hline Infusion mode, bolus/continuous & $87 / 91$ & $72 / 69$ & $15 / 22$ & 0.25 \\
\hline Symptoms, moderate/severe & $134 / 44$ & $114 / 27$ & $20 / 17$ & 0.001 \\
\hline \multicolumn{5}{|l|}{ Medication } \\
\hline Potassium sparing diuretics & $6(3.4)$ & $6(4.3)$ & $0(0)$ & 0.35 \\
\hline Furosemide & $13(7.3)$ & $11(7.8)$ & $2(5.4)$ & $>0.99$ \\
\hline SSRI & $13(7.3)$ & $8(5.7)$ & $5(13.5)$ & 0.15 \\
\hline AED & $22(12.4)$ & $14(9.9)$ & $8(21.6)$ & 0.09 \\
\hline NSAID & $42(23.6)$ & $33(23.4)$ & $9(24.3)$ & 0.91 \\
\hline Systolic BP (mmHg) & $139.2 \pm 25.3$ & $138.3 \pm 25.2$ & $142.6 \pm 25.7$ & 0.53 \\
\hline Diastolic BP (mmHg) & $75.7 \pm 13.8$ & $75.2 \pm 13.0$ & $77.4 \pm 16.8$ & 0.33 \\
\hline \multicolumn{5}{|l|}{ Laboratory values } \\
\hline $\mathrm{Na}(\mathrm{mEq} / \mathrm{L})$ & $118.2 \pm 5.0$ & $119.2 \pm 4.2$ & $114.5 \pm 6.0$ & $<0.001$ \\
\hline Serum osmolality (mOsm/kg) & $251.1 \pm 21.6$ & $252.2 \pm 16.3$ & $246.6 \pm 35.1$ & $<0.001$ \\
\hline Creatinine $(\mathrm{mg} / \mathrm{dL})$ & $1.0 \pm 0.8$ & $1.0 \pm 0.9$ & $0.8 \pm 0.3$ & 0.08 \\
\hline White blood cell $\left(\times 10^{9} / \mathrm{L}\right)$ & $8.5 \pm 4.0$ & $8.4 \pm 3.8$ & $9.2 \pm 4.9$ & 0.34 \\
\hline Hemoglobin $(\mathrm{g} / \mathrm{dL})$ & $12.1 \pm 2.0$ & $12.0 \pm 2.0$ & $12.3 \pm 1.7$ & 0.48 \\
\hline Albumin (g/dL) & $4.0 \pm 0.6$ & $3.9 \pm 0.6$ & $4.2 \pm 0.6$ & 0.02 \\
\hline Calcium (mg/dL) & $8.7 \pm 0.5$ & $8.7 \pm 0.5$ & $8.7 \pm 0.5$ & 0.76 \\
\hline Phosphorous (mg/dL) & $3.1 \pm 0.9$ & $3.1 \pm 1.0$ & $2.7 \pm 0.6$ & 0.003 \\
\hline Potassium (mEq/L) & $4.0 \pm 0.8$ & $4.1 \pm 0.7$ & $3.6 \pm 0.7$ & $<0.001$ \\
\hline Total $\mathrm{CO}_{2}(\mathrm{mEq} / \mathrm{L})$ & $23.2 \pm 5.0$ & $23.2 \pm 5.3$ & $23.0 \pm 3.8$ & 0.59 \\
\hline Total cholesterol (mg/dL) & $155.3 \pm 44.7$ & $154.8 \pm 41.8$ & $157.4 \pm 54.8$ & 0.79 \\
\hline Total bilirubin (mg/dL) & $0.9 \pm 0.5$ & $0.9 \pm 0.4$ & $1.1 \pm 0.6$ & 0.003 \\
\hline AST (units/L) & $35.0 \pm 29.2$ & $32.7 \pm 27.2$ & $43.7 \pm 34.8$ & 0.01 \\
\hline ALT (units/L) & $20.2 \pm 17.0$ & $19.1 \pm 17.7$ & $24.5 \pm 13.8$ & 0.001 \\
\hline C-reactive protein (mg/L) & $28.3 \pm 48.5$ & $30.0 \pm 50.3$ & $21.9 \pm 40.8$ & 0.21 \\
\hline Urine osmolality (mOsm/kg) & $424.1 \pm 164.2$ & $424.7 \pm 170.4$ & $421.9 \pm 139.9$ & 0.85 \\
\hline Urine $\mathrm{Na}(\mathrm{mEq} / \mathrm{L})$ & $72.1 \pm 49.3$ & $71.8 \pm 48.7$ & $73.2 \pm 52.3$ & 0.98 \\
\hline Urine $\mathrm{K}(\mathrm{mEq} / \mathrm{L})$ & $34.6 \pm 22.5$ & $34.2 \pm 23.5$ & $36.1 \pm 18.8$ & 0.32 \\
\hline \multicolumn{5}{|c|}{ Cumulative amount of $3 \%$ saline volume $(\mathrm{mL})$} \\
\hline $1 \mathrm{hr}$ & $127.4 \pm 107.6$ & $128.9 \pm 105.4$ & $121.7 \pm 116.8$ & 0.68 \\
\hline $6 \mathrm{hr}$ & $241.5 \pm 115.4$ & $247.3 \pm 112.5$ & $219.1 \pm 125.1$ & 0.14 \\
\hline $24 \mathrm{hr}$ & $402.5 \pm 257.3$ & $416.3 \pm 244.0$ & $350.0 \pm 300.6$ & 0.05 \\
\hline $48 \mathrm{hr}$ & $554 \pm 347.9$ & $598.1 \pm 337.2$ & $388.5 \pm 342.2$ & 0.001 \\
\hline Urine volume during $48 \mathrm{hr}(\mathrm{mL})$ & $3,862 \pm 2,830$ & $3,401 \pm 2,610$ & $5,653 \pm 2,973$ & $<0.001$ \\
\hline
\end{tabular}

Data are expressed as number only, number (\%), or mean \pm standard deviation.

AED, antiepileptic drug; ALT, alanine aminotransferase; AST, aspartate transaminase; BP, blood pressure; d/t, due to; ECF, extracellular fluid; Na, sodium; NSAID, nonsteroidal anti-inflammatory drug; SIAD, syndrome of inappropriate antidiuresis; SSRI, selective serotonin reuptake inhibitor. 
Table 2. Risk factors for overcorrection by univariable and multivariable logistic regression

\begin{tabular}{|c|c|c|c|c|}
\hline \multirow{2}{*}{ Variable } & \multicolumn{2}{|c|}{ Univariable analysis } & \multicolumn{2}{|c|}{ Multivariable ${ }^{a}$ analysis } \\
\hline & OR & p-value & OR & p-value \\
\hline \multicolumn{5}{|l|}{ Demographics } \\
\hline Male sex & 0.80 & 0.55 & & \\
\hline Age & 0.98 & 0.15 & & \\
\hline Weight & 1.01 & 0.38 & & \\
\hline \multicolumn{5}{|l|}{ Cause of hyponatremia } \\
\hline Decreased ECF d/t nonrenal Na loss & 0.80 & 0.62 & & \\
\hline Increased ECF & 0.42 & 0.26 & & \\
\hline SIAD & 0.73 & 0.46 & & \\
\hline Thiazide use & 2.14 & 0.05 & & \\
\hline Adrenal insufficiency & 0.81 & 0.62 & & \\
\hline \multicolumn{5}{|l|}{ Comorbidity } \\
\hline Diabetes mellitus & 1.12 & 0.77 & & \\
\hline Hypertension & 2.21 & 0.08 & & . \\
\hline Malignancy & 0.71 & 0.45 & & \\
\hline Chronic alcoholism & 6.13 & 0.05 & 15.27 & 0.02 \\
\hline Infusion mode, bolus/continuous & 1.53 & 0.26 & & \\
\hline Symptoms, moderate/severe & 3.59 & $<0.001$ & 2.87 & 0.03 \\
\hline \multicolumn{5}{|l|}{ Laboratory values } \\
\hline $\mathrm{Na}$ & 0.84 & $<0.001$ & 0.86 & 0.002 \\
\hline Potassium & 0.37 & 0.001 & 0.34 & 0.001 \\
\hline Creatinine & 0.50 & 0.13 & & \\
\hline White blood cell & 1.05 & 0.26 & & \\
\hline Hemoglobin & 1.07 & 0.49 & & \\
\hline Albumin & 2.38 & 0.02 & & \\
\hline Total bilirubin & 3.06 & 0.004 & & \\
\hline AST & 1.01 & 0.08 & & \\
\hline ALT & 1.02 & 0.13 & & \\
\hline C-reactive protein & 0.96 & 0.37 & & \\
\hline Urine osmolality & 1.00 & 0.93 & & \\
\hline
\end{tabular}

ALT, alanine aminotransferase; AST, aspartate transaminase; d/t, due to; ECF, extracellular fluid; Na, sodium; SIAD, syndrome of inappropriate antidiuresis; SSRI, selective serotonin reuptake inhibitor;

${ }^{a}$ Age, sex, body weight, cause of hyponatremia, hypertension, diabetes mellitus, cancer, chronic alcoholics, infusion mode, symptoms, serum Na, potassium, white blood cell, hemoglobin, albumin, total bilirubin, AST, ALT, C-reactive protein, urine osmolality.

using risk factors for overcorrection based on multivariable analysis as described in Supplementary Table 1 (available online). The NASK (hypoNatremia, Alcoholism, Severe symptoms, and hypoKalemia) score was calculated as the arithmetic sum of the points for each of these variables. The scores were as follows: chronic alcoholism, 7 points; severe symptoms of hyponatremia, 3 points; low serum potassium level of $<3.0 \mathrm{mEq} / \mathrm{L}, 3$ points; and initial sodium level of $\leq 110,110-115,115-120$, and 120-125 mEq/L were scored as 7, 4, 2, and 0 points, respectively. NASK score was significantly associated with overcorrection (OR, 1.41;
95\% CI, 1.24-1.61; $\mathrm{p}<0.01$ ) (Fig. 1). The receiver-operating characteristic curves for each risk factor and NASK score with regard to overcorrection are shown in Fig. 2. NASK score had a good discriminatory ability, with an AUROC curve of 0.757 (95\% CI, 0.66-0.85; $\mathrm{p}<0.01$ ). The AUROC was 0.752 (95\% CI, 0.67-0.84; $\mathrm{p}=0.88$ ), 0.691 (95\% CI, 0.60 $0.79 ; \mathrm{p}=0.27$ ), 0.634 (95\% CI, 0.55-0.72; $<<0.01$ ), and 0.533 (95\% CI, 0.49-0.58; $\mathrm{p}<0.01$ ) for the baseline serum sodium level, baseline serum potassium level, severity of hyponatremia symptoms, and chronic alcoholism, respectively. The AUROC curve of the NASK score was significantly bet- 
ter than that of each risk factor.

Comparison of the predictive abilities of NASK and SHOR scores

We compared the predictive ability of NASK and SHOR1/ SHOR2 scores for hyponatremia overcorrection using

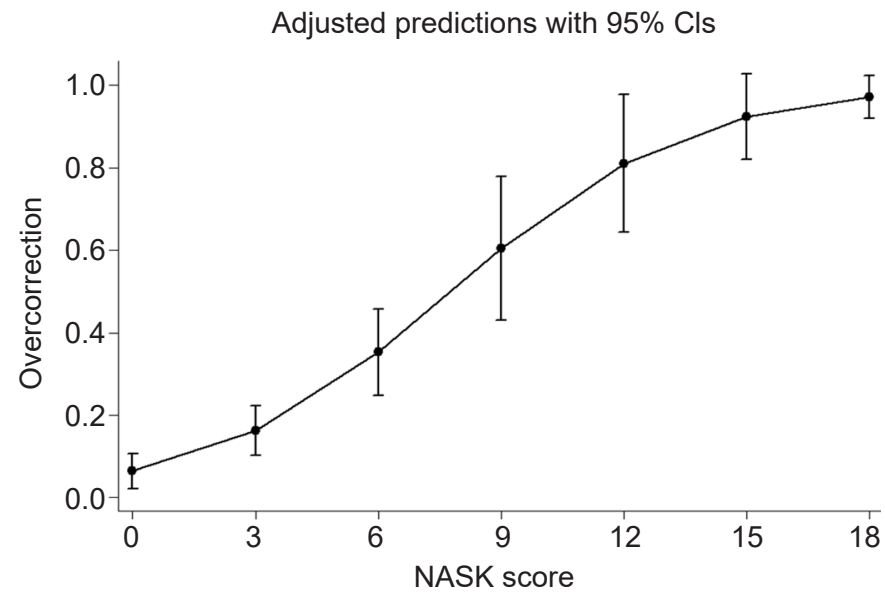

Figure 1. Marginal plot of the NASK score against hyponatremia overcorrection.

$\mathrm{Cl}$, confidence interval; NASK, hypoNatremia, Alcoholism, Severe symptoms, and hypoKalemia.
AUROC curve analysis (Supplementary Fig. 2, available online). The AUROC curve were 0.757 (95\% CI, 0.66-0.85; $\mathrm{p}<0.01$ ), 0.722 (95\% CI, 0.63-0.82; p = 0.46), 0.722 (95\% CI, 0.63-0.82; $\mathrm{p}=0.47$ ) for NASK, SHOR1, and SHOR2 scores, respectively. The AUROC curve for NASK score was higher than those of SHOR1 and SHOR2 scores, although there were no significant differences between the values.

\section{Cumulative hyponatremia overcorrection and predictive ability of NASK score by time}

We subdivided the overcorrection group by time (within the first $6,12,24$, and 48 hours) to evaluate whether hyponatremia overcorrection risk factors changed according to time. Overcorrection occurred in $5.1 \%(\mathrm{n}=9), 6.7 \%(\mathrm{n}$ $=12), 18.5 \%(\mathrm{n}=33)$, and $20.8 \%(\mathrm{n}=37)$ of 178 patients within the first $6,12,24$, and 48 hours, respectively. We analyzed the predictive ability of the four risk factors for overcorrection and NASK score by time based on age, sex, and hypertonic saline infusion method. The four identified risk factors (initial serum sodium level, chronic alcoholism, initial symptoms, and initial serum potassium level) and NASK score had significant predictive abilities for cumulative overcorrection within $6,12,24$, and 48 hours, excluding initial potassium level within 6 hours and chronic alcohol-

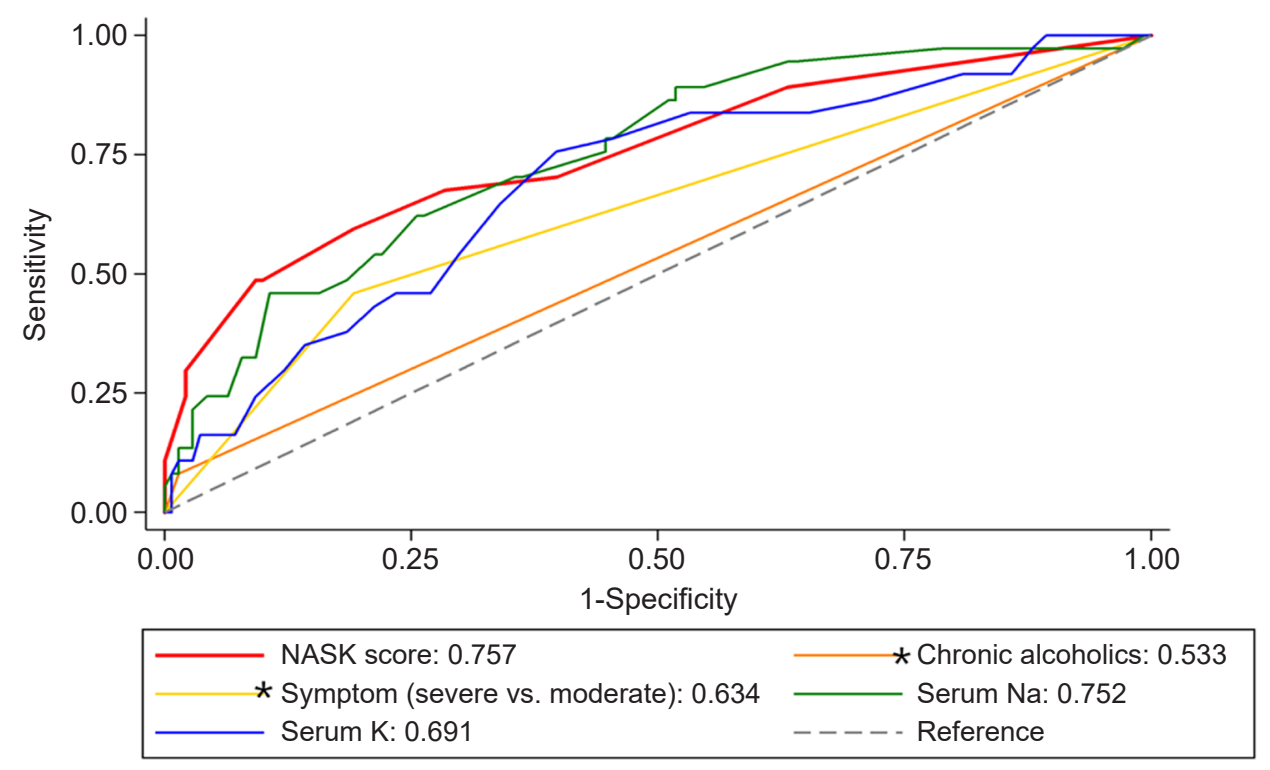

Figure 2. The AUROC curve for the NASK score and each risk factor for hyponatremia overcorrection. The AUROC curve for each of the two factors (*) is statistically significant when compared to the AUROC of NASK score.

AUROC, the area under the receiver-operating characteristic; NASK, hypoNatremia, Alcoholism, Severe symptoms, and hypoKalemia. 


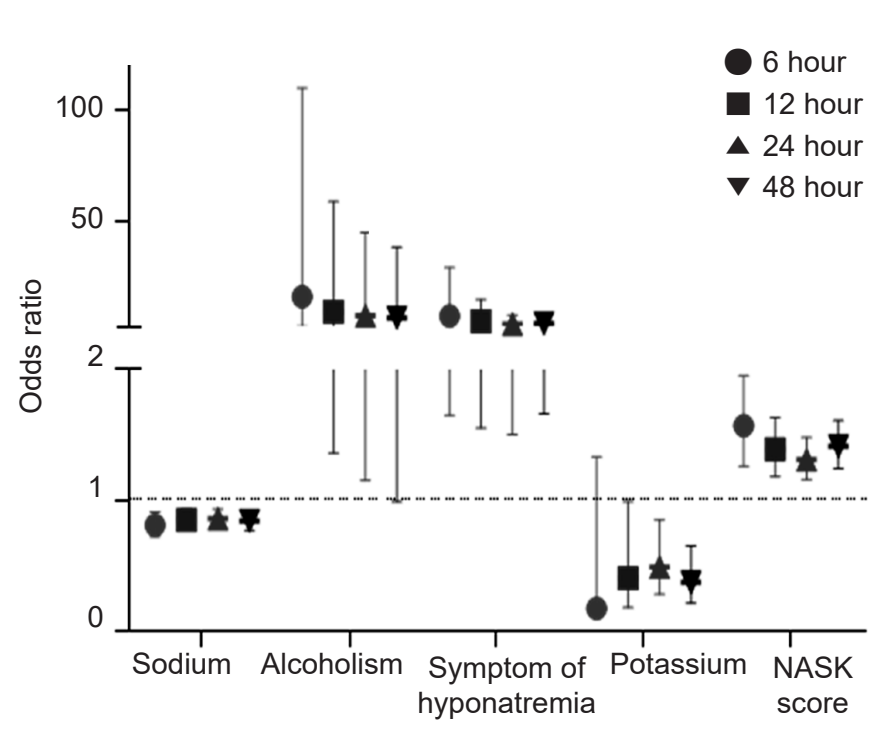

Figure 3. Unadjusted odds ratios of the risk factors for hyponatremia overcorrection.

NASK, hypoNatremia, Alcoholism, Severe symptoms, and hypoKalemia.

ism within 48 hours (Fig. 3, Fig. 4; Supplementary Table 2, available online).

Urine output over 48 hours based on hyponatremia overcorrection risk factors

The 48 hours urine output was higher in chronic alcoholics than in non-alcoholics (5,688 $\mathrm{mL}$ vs. $3,809 \mathrm{~mL}, \mathrm{p}=0.08)$, although the difference was not significant. Furthermore, the urine output was higher in patients with severe symptoms than that of patients with moderate symptoms $(5,231 \mathrm{~mL}$ vs. $3,419 \mathrm{~mL}, \mathrm{p}<0.01)$. Patients with severe hyponatremia had a higher urine output than in those with less severe hyponatremia $(6,025,4,158,3,767,3,378 \mathrm{~mL}$ for patients with serum sodium levels of $\leq 110,110-115,115-120$, and $\geq 120$ $\mathrm{mEq} / \mathrm{L}$, respectively; $\mathrm{p}=0.02$ ). Moreover, there was a negative correlation between hypokalemia and urine output based on linear regression analysis $(\beta=-0.216, p<0.01)$. NASK score also was positively associated with 48 hours urine output $(\beta=304.5, \mathrm{p}<0.001)$.

\section{Discussion}

In this post hoc analysis of a prospective randomized con-

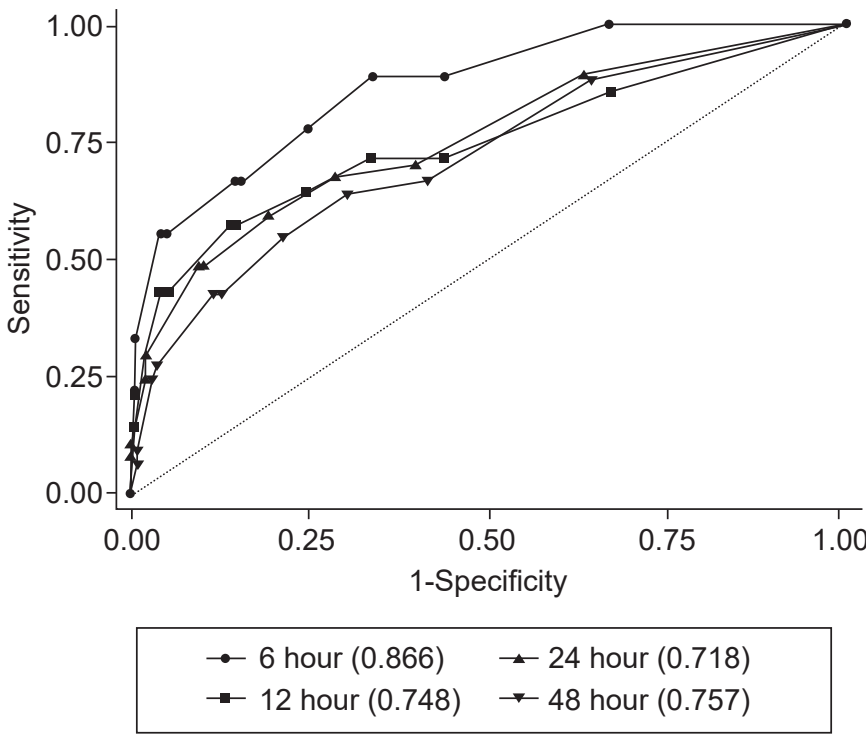

Figure 4. Cumulative hyponatremia overcorrection and the predictive ability of the NASK score by time.

NASK, hypoNatremia, Alcoholism, Severe symptoms, and hypoKalemia.

trolled study, we aimed to evaluate risk factors for hyponatremia overcorrection and to establish a novel scoring system for predicting overcorrection. We identified chronic alcoholism and severe symptoms of hyponatremia as well as lower baseline serum sodium and potassium levels as significant risk factors for hyponatremia overcorrection. The NASK score, an arithmetic sum of the points for each factor after converting the influence of each factor to integer scores, had a higher predictive ability for hyponatremia overcorrection than each factor. Chronic alcoholism, severe symptoms of hyponatremia, low baseline serum sodium levels, and NASK score were risk factors for overcorrection at any time within 48 hours. However, low baseline serum potassium was a risk factor for overcorrection only after 6 hours.

We found that chronic alcoholism, initial serum sodium level, severity of initial symptoms, and initial serum potassium level were significant patient baseline factors that affected the incidence of hyponatremia overcorrection. Alcohol suppresses the endogenous release of antidiuretic hormone $(\mathrm{ADH})$ and occasionally causes free water diuresis when consumed. However, continuous alcohol consumption increases ADH levels, thereby causing water retention. Water and electrolyte retention are resolved 
within 3 to 6 days after alcohol discontinuation in chronic alcoholics [28,29]. When chronic alcoholics are admitted for hyponatremia, they undergo a period of alcohol withdrawal. Therefore, chronic alcoholics have an increased overcorrection risk during hypertonic saline treatment due to increased diuresis as confirmed by our findings. Previous studies have revealed that lower initial sodium levels $[14,17,18,21]$ and severe symptoms of hyponatremia $[17,21]$ are risk factors for hyponatremia overcorrection, in line with our finding. Hyponatremia overcorrection mainly arises from hypertonic saline treatment or water diuresis. Hyponatremia causes a hypo-osmolar state, leading to a decreased release of $\mathrm{ADH}$ and an increase in free water excretion. Supplying hypertonic saline in patients with hyponatremia induces an increase in $\mathrm{ADH}$ level and a decrease in water clearance, which occur at a slower rate in patients with severe hyponatremia [30]. However, we cannot explain the mechanism underlying free water excretion using our data because we did not collect urine sodium and potassium levels, as well as osmolality during the 48-hour follow-up. Symptoms of hyponatremia were classified into two groups, and the initial hypertonic saline infusion rate was decided based on symptom severity. A higher amount of hypertonic saline was administered in patients with severe symptoms compared with that in patients with moderate symptoms $(317 \mathrm{~mL}$ vs. $217 \mathrm{~mL}, \mathrm{p}<$ 0.01 ; within the first 6 hours). In line with previous studies $[14,17]$, we found that lower initial potassium levels were associated with hyponatremia overcorrection occurrence. In the Adrogue-Madias formula, replacing potassium plays a significant role in correcting hyponatremia $[7,31]$. The loss of sodium or potassium induces an osmolar shift to maintain the osmolar balance between the extracellular and intracellular spaces [32]. Potassium loss shifts sodium intracellularly, induces hyponatremia, and enhances $\mathrm{ADH}$ release, thereby worsening hyponatremia [33]. Moreover, potassium restriction reportedly increases free water clearance [34]. Therefore, hypokalemia can increase the incidence of hyponatremia overcorrection by increasing diuresis, as confirmed by our findings. Not only each factor but also the NASK score, which combines the scores of these factors, had a positive relationship with the 48 hours urine output.

In addition to the four risk factors identified in our study, previous studies have identified younger age, higher infu- sion volume, lower urine osmolality, and lower urine sodium levels as risk factors for hyponatremia overcorrection $[14,21]$. Volume overload and the presence of chest tumor are negatively associated with hyponatremia overcorrection [17]. Woodfine et al. [17] established a novel scoring system (the SHOR score) to predict hyponatremia overcorrection, with risk factors such as age, vomiting, somnolence, volume overload, initial serum sodium level, initial serum potassium level, urine osmolality, and the presence of chest tumor. Our study found the following risk factors for hyponatremia overcorrection: chronic alcoholism, initial serum sodium level, severity of initial symptoms, and initial serum potassium level. Moreover, we developed a scoring system (NASK score) to aid clinicians in quantitatively stratifying an individual's risk for hyponatremia overcorrection. We compared the predictive ability of hyponatremia overcorrection between the NASK scoring system and the SHOR scoring system [17]. The AUROC curve for NASK score was greater than those for SHOR1 and SHOR2 scores but without statistical significance (Supplementary Fig. 2). Nevertheless, NASK score is easy to calculate as it requires only four factors, whereas SHOR scores require eight discrete factors. Furthermore, NASK score seems to predict hyponatremia overcorrection better than SHOR scores.

This study has several strengths. First, we obtained complete baseline characteristics as well as laboratory data on all patients because of the prospective nature of the original study. We also assessed the cumulative hyponatremia overcorrection rate and verified the predictive ability of each risk factor and the NASK score for the overcorrection rate. Second, in comparison with other retrospective studies, we set up the treatment protocol with hypertonic saline and serum sodium relowering treatment following international guideline recommendations (RIB and SCI). This allowed us to correct the impact of the infusion method of hypertonic saline on treatment outcomes. Therefore, we were able to establish a more explainable scoring system using baseline characteristics. There are several studies or guidelines regarding the adequate amount of hypertonic saline and the required rate for effective hyponatremia treatment and hyponatremia overcorrection prevention. However, inadvertent hyponatremia overcorrection occurs because of unanticipated water diuresis, even when the recommended quantity of hypertonic saline is administrated at a recommended rate or when hypertonic saline administration is 
stopped. Using this scoring system, physicians can predict hyponatremia overcorrection occurrence before hypertonic saline administration; patients with high NASK scores and increased diuresis can undergo careful monitoring of their status and laboratory data during treatment.

This study also has several limitations. First, due to the prospective design, our study population was smaller than those of other retrospective studies. Second, serum sodium levels were obtained from the three study sites using different measuring machines that were not calibrated. Variabilities in serum sodium assays using each device were inevitable due to the post hoc study design. Third, low sensitivity and positive predictive value were imperative, as the prevalence of chronic alcoholism was low. We additionally performed sensitivity analyses with the NSK (Na, symptoms of hyponatremia, and K) score (total, $\leq 13$ ) and NK ( Na and K) score (total, $\leq 10$ ) for hyponatremia overcorrection models. The AUROC curves for NSK and NK scores were 0.745 (95\% CI, 0.65-0.84; $\mathrm{p}<0.01$ ) and 0.721 (95\% CI, 0.63-0.81; $\mathrm{p}<$ 0.01 ), respectively. Fourth, the findings were not validated in an external cohort of patients with hyponatremia. A validation study with a larger population should be conducted to confirm our study findings. Specifically, further studies should clarify whether treatment policies should be modified based on the finding that the NASK score influences hyponatremia overcorrection incidence and prognosis. Fifth, some studies have reported a relationship between the occurrences of hyponatremia overcorrection and ODS, which is a critical outcome of hyponatremia overcorrection $[11,14,17,24,35,36]$. However, there was no ODS in $20.8 \%$ of patients with hyponatremia overcorrection, although risk factors for overcorrection were identified to prevent ODS in patients with hyponatremia by reducing overcorrection occurrence. Hyponatremia overcorrection is considered a good laboratory outcome that predicts ODS occurrence because it can be monitored. Moreover, it is a correctable factor during hypertonic saline treatment.

In conclusion, hyponatremia overcorrection occurred in $20.8 \%$ of patients who underwent hypertonic saline treatment. The risk factors for hyponatremia overcorrection included chronic alcoholism and severe symptoms of hyponatremia as well as lower initial serum sodium and potassium levels. In patients undergoing symptomatic hyponatremia treatment, overcorrection might be quantitatively predicted using a novel risk score that is summarized by patient baseline information. External validation studies of the NASK score are required to clarify our results.

\section{Conflicts of interest}

All authors have no conflicts of interest to declare.

\section{Funding}

The study was supported by grants from the National Research Foundation of Korea (No. 2021R1C1C1008966 and 2019R1A2C1085411).

\section{Authors' contributions}

Conceptualization: HY, SHB, SK

Data curation: SHB, SK

Formal analysis: HY, SHB, SK

Funding acquisition: SHB, SK

Technical and material support: All authors

Visualization: HY, SHB, SK

Writing-original draft: HY, SHB, SK

Writing-review \& editing: All authors

All authors read and approved the final manuscript.

\section{ORCID}

Huijin Yang, https://orcid.org/0000-0001-5885-1519

Songuk Yoon, https://orcid.org/0000-0002-9029-1918

Eun Jung Kim, https://orcid.org/0000-0002-4033-2769

Jang Won Seo, https://orcid.org/0000-0002-3495-5388

Ja-Ryong Koo, https://orcid.org/0000-0003-4245-2569

Yun Kyu Oh, https://orcid.org/0000-0001-8632-5743

You Hwan Jo, https://orcid.org/0000-0002-9507-7603

Sejoong Kim, https://orcid.org/0000-0002-7238-9962

Seon Ha Baek, https://orcid.org/0000-0002-4751-9817

\section{References}

1. Spasovski G, Vanholder R, Allolio B, et al. Clinical practice guideline on diagnosis and treatment of hyponatraemia. Nephrol Dial Transplant 2014;29 Suppl 2:i1-i39.

2. Upadhyay A, Jaber BL, Madias NE. Incidence and prevalence of hyponatremia. Am J Med 2006;119(7 Suppl 1):S30-S35.

3. Decaux G. Is asymptomatic hyponatremia really asymptomatic? 
Am J Med 2006;119(7 Suppl 1):S79-S82.

4. Nigro N, Winzeler B, Suter-Widmer I, et al. Symptoms and characteristics of individuals with profound hyponatremia: a prospective multicenter observational study. J Am Geriatr Soc 2015;63:470-475.

5 . Knochel JP. Hypoxia is the cause of brain damage in hyponatremia. JAMA 1999;281:2342-2343.

6. Ayus JC, Varon J, Arieff AI. Hyponatremia, cerebral edema, and noncardiogenic pulmonary edema in marathon runners. Ann Intern Med 2000;132:711-714.

7. Adrogué HJ, Madias NE. Hyponatremia. N Engl J Med 2000; 342:1581-1589.

8. Gross P, Reimann D, Neidel J, et al. The treatment of severe hyponatremia. Kidney Int Suppl 1998;64:S6-S11.

9. Ayus JC, Olivero JJ, Frommer JP. Rapid correction of severe hyponatremia with intravenous hypertonic saline solution. Am J Med 1982;72:43-48.

10. Mohmand HK, Issa D, Ahmad Z, Cappuccio JD, Kouides RW, Sterns RH. Hypertonic saline for hyponatremia: risk of inadvertent overcorrection. Clin J Am Soc Nephrol 2007;2:1110-1117.

11. Sterns RH, Riggs JE, Schochet SS Jr. Osmotic demyelination syndrome following correction of hyponatremia. $N$ Engl J Med 1986;314:1535-1542.

12. Verbalis JG, Goldsmith SR, Greenberg A, Schrier RW, Sterns RH. Hyponatremia treatment guidelines 2007: expert panel recommendations. Am J Med 2007;120(11 Suppl 1):S1-S21.

13. Verbalis JG, Goldsmith SR, Greenberg A, et al. Diagnosis, evaluation, and treatment of hyponatremia: expert panel recommendations. Am J Med 2013;126(10 Suppl 1):S1-S42.

14. George JC, Zafar W, Bucaloiu ID, Chang AR. Risk factors and outcomes of rapid correction of severe hyponatremia. Clin J Am Soc Nephrol 2018;13:984-992.

15. Sterns RH, Hix JK. Overcorrection of hyponatremia is a medical emergency. Kidney Int 2009;76:587-589.

16. Woodfine JD, van Walraven C. Criteria for hyponatremic overcorrection: systematic review and cohort study of emergently ill patients. J Gen Intern Med 2020;35:315-321.

17. Woodfine JD, Sood MM, MacMillan TE, Cavalcanti RB, van Walraven C. Derivation and validation of a novel risk score to predict overcorrection of severe hyponatremia: the Severe Hyponatremia Overcorrection Risk (SHOR) Score. Clin J Am Soc Nephrol 2019;14:975-982.

18. Aratani S, Hara M, Nagahama M, et al. A low initial serum sodium level is associated with an increased risk of overcorrection in patients with chronic profound hyponatremia: a retrospective cohort analysis. BMC Nephrol 2017;18:316.

19. Lee A, Jo YH, Kim K, et al. Efficacy and safety of rapid intermittent correction compared with slow continuous correction with hypertonic saline in patients with moderately severe or severe symptomatic hyponatremia: study protocol for a randomized controlled trial (SALSA trial). Trials 2017;18:147.

20. Baek SH, Jo YH, Ahn S, et al. Risk of overcorrection in rapid intermittent bolus vs slow continuous infusion therapies of hypertonic saline for patients with symptomatic hyponatremia: the SALSA Randomized Clinical Trial. JAMA Intern Med 2021;181:81-92.

21. Geoghegan P, Harrison AM, Thongprayoon C, et al. Sodium correction practice and clinical outcomes in profound hyponatremia. Mayo Clin Proc 2015;90:1348-1355.

22. Filippatos TD, Liamis G, Elisaf MS. Ten pitfalls in the proper management of patients with hyponatremia. Postgrad Med 2016;128:516-522.

23. Moritz ML, Ayus JC. 100 cc 3\% sodium chloride bolus: a novel treatment for hyponatremic encephalopathy. Metab Brain Dis 2010;25:91-96.

24. Sterns RH, Cappuccio JD, Silver SM, Cohen EP. Neurologic sequelae after treatment of severe hyponatremia: a multicenter perspective. J Am Soc Nephrol 1994;4:1522-1530.

25. Hillier TA, Abbott RD, Barrett EJ. Hyponatremia: evaluating the correction factor for hyperglycemia. Am J Med 1999;106:399403.

26. Fenske W, Störk S, Blechschmidt A, Maier SG, Morgenthaler NG, Allolio B. Copeptin in the differential diagnosis of hyponatremia. J Clin Endocrinol Metab 2009;94:123-129.

27. Donato AA, Lloyd BJ. In hyponatremia, rapid intermittent bolus vs. slow continuous infusion of hypertonic saline did not differ for overcorrection of serum sodium. Ann Intern Med 2021;174:JC33.

28. Adewale A, Ifudu O. Kidney injury, fluid, electrolyte and acid-base abnormalities in alcoholics. Niger Med J 2014;55:93-98.

29. Ragland G. Electrolyte abnormalities in the alcoholic patient. Emerg Med Clin North Am 1990;8:761-773.

30. Jensen JM, Mose FH, Bech JN, Nielsen S, Pedersen EB. Effect of volume expansion with hypertonic- and isotonic saline and isotonic glucose on sodium and water transport in the principal cells in the kidney. BMC Nephrol 2013;14:202.

31. Berl T, Rastegar A. A patient with severe hyponatremia and hypokalemia: osmotic demyelination following potassium repletion. Am J Kidney Dis 2010;55:742-748.

32. Heppel LA. The electrolytes of muscle and liver in potassium-de- 
pleted rats. Am J Physiol Leg Content 1939;127:385-392.

33. Schrier RW, Berl T, Anderson RJ. Osmotic and nonosmotic control of vasopressin release. Am J Physiol 1979;236:F321-F332.

34. Gallen IW, Rosa RM, Esparaz DY, et al. On the mechanism of the effects of potassium restriction on blood pressure and renal sodium retention. Am J Kidney Dis 1998;31:19-27.
35. Karp BI, Laureno R. Pontine and extrapontine myelinolysis: a neurologic disorder following rapid correction of hyponatremia. Medicine (Baltimore) 1993;72:359-373.

36. Sterns RH, Nigwekar SU, Hix JK. The treatment of hyponatremia. Semin Nephrol 2009;29:282-299. 